\title{
The Cell Ontology 2016: enhanced content, modularization, and ontology interoperability
}

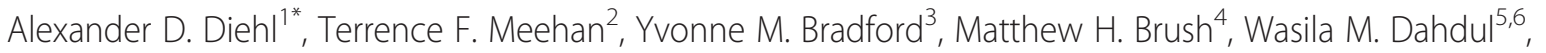 \\ David S. Dougall7, Yongqun He ${ }^{8}$, David Osumi-Sutherland ${ }^{2}$, Alan Ruttenberg ${ }^{9}$, Sirarat Sarntivijai ${ }^{2}$, Ceri E. Van Slyke ${ }^{3}$, \\ Nicole A. Vasilevsky ${ }^{4}$, Melissa A. Haendel ${ }^{4}$, Judith A. Blake ${ }^{10}$ and Christopher J. Mungall ${ }^{11}$
}

\begin{abstract}
Background: The Cell Ontology $(\mathrm{CL})$ is an OBO Foundry candidate ontology covering the domain of canonical, natural biological cell types. Since its inception in 2005, the CL has undergone multiple rounds of revision and expansion, most notably in its representation of hematopoietic cells. For in vivo cells, the $C L$ focuses on vertebrates but provides general classes that can be used for other metazoans, which can be subtyped in species-specific ontologies.

Construction and content: Recent work on the $\mathrm{CL}$ has focused on extending the representation of various cell types, and developing new modules in the CL itself, and in related ontologies in coordination with the CL. For example, the Kidney and Urinary Pathway Ontology was used as a template to populate the CL with additional cell types. In addition, subtypes of the class 'cell in vitro' have received improved definitions and labels to provide for modularity with the representation of cells in the Cell Line Ontology and Reagent Ontology. Recent changes in the ontology development methodology for $\mathrm{CL}$ include a switch from $\mathrm{OBO}$ to $\mathrm{OWL}$ for the primary encoding of the ontology, and an increasing reliance on logical definitions for improved reasoning.

Utility and discussion: The $C L$ is now mandated as a metadata standard for large functional genomics and transcriptomics projects, and is used extensively for annotation, querying, and analyses of cell type specific data in sequencing consortia such as FANTOM5 and ENCODE, as well as for the NIAID ImmPort database and the Cell Image Library. The $\mathrm{CL}$ is also a vital component used in the modular construction of other biomedical ontologies-for example, the Gene Ontology and the cross-species anatomy ontology, Uberon, use CL to support the consistent representation of cell types across different levels of anatomical granularity, such as tissues and organs.

Conclusions: The ongoing improvements to the $\mathrm{CL}$ make it a valuable resource to both the $\mathrm{OBO}$ Foundry community and the wider scientific community, and we continue to experience increased interest in the $C L$ both among developers and within the user community.
\end{abstract}

\footnotetext{
* Correspondence: addiehl@buffalo.edu

${ }^{1}$ Department of Neurology, University at Buffalo School of Medicine and

Biomedical Sciences, Buffalo, NY 14203, USA

Full list of author information is available at the end of the article
} 


\section{Background}

The Cell Ontology (CL) was initially developed in 2004 with the goal of representing knowledge about in vivo and in vitro cell types [1]. Cells are a fundamental unit of biology, and most other entities in biology have direct relationships to identifiable cell types, for example particular proteins being produced by unique cell types, tissues and organs containing specific combinations of cell types, or biological processes being dependent on particular cell types. Cells therefore are an obvious set of entities to represent ontologically, and provide a useful pole for organizing and driving data acquisition and analysis in biology.

The content in the CL is populated via gradual and en masse class additions, most notably through several rounds of improvements to representation of hematopoietic cells in the ontology [2-4]. Originally, the CL was designed to include cell types from all major model organisms including both plants and animals [1]. However, as a result of community interest and severe resource limitations, continuing development of the CL currently focuses primarily on vertebrate cell types. The CL provides general classes that can be used for other metazoans (muscle cell, neuron), and the ontology can be extended in species-specific ontologies.

The CL is built according to the principles established by the OBO Foundry [5] and is the designated candidate ontology for metazoan cell types within the Foundry. The domain and content of CL is intended to be orthogonal to other Foundry ontologies to allow for the construction of compositional classes via logical definitions, as exemplified by the Gene Ontology (GO) [3, 6-8].

Work on the CL over the past several years has resulted in many improvements in the ontology's structure and content. As described below, cooperation among a number of working groups has resulted in a modular approach to improving the $\mathrm{CL}$, and continued enhancement of logical definitions in the CL have increased its integration and interoperability with other ontologies as well as enhancing its utility for data analysis.

\section{Construction and content}

\section{Editorial management of the $\mathrm{CL}$}

The CL is maintained primarily by a small group of editors (ADD, YB, MH, DOS, CVS, NV, CJM), working in conjunction with interested parties from the ontology community. The editors use biweekly teleconferences to discuss significant issues related to CL ontology development. Because the CL has not been directly funded in recent years, most efforts are contributed as part of other projects and reflect the cooperative efforts of ontology developers and users based in different communities, such as the Gene Ontology Consortium [8, 9], the Immunology Database and Analysis Portal (ImmPort)
[10], the Human Immunology Project Consortium (HIPC) [11], the Phenoscape project [12, 13], the Monarch Initiative [14], and model organism databases such as the Zebrafish Model Organism database (ZFIN) [15] and Mouse Genome Informatics (MGI) [16]. Consequently term creation occurs at an uneven pace, based on requests and editor availability. Over the past few years, we have received approximately 3-5 term requests per month. Most requests are accommodated in 1-3 months. The $\mathrm{CL}$ is released on an ad hoc basis, with new releases 5-6 times per year.

We welcome involvement of the community on particular domain specific developments, as has been done with kidney cell types (see below) and with immune cell types through our continuing collaboration with HIPC. Collaboration with the larger biological and biomedical community occurs both through our issue tracker and through direct contacts with any of the editors.

\section{Cell types in CL}

As of June 2016, The CL contains approximately 2,200 classes, compared with 1534 at the time of our last report [3].The relative distribution of number of cell types among categories remains relatively constant, with one of the most well-represented being the hematopoietic cell branch, as described in [2-4], currently totaling 575 classes. Although the size of this branch has remained relatively constant, the content is continually refined and improved. For example, many of the original hematopoietic cell definitions are being reviewed and generalized to be applicable beyond mouse and human.

One area of expansion has been kidney cell subtypes, resulting from collaboration with the Kidney and Urinary Pathway Ontology (KUPO) project [17] as well as the Gene Ontology [18]. This has resulted in the addition of 125 new classes to represent kidney cell subtypes.

Over 400 cell types were added by generalizing human-specific classes from the Foundation Model of Anatomy (FMA) [19, 20] -many of these were compositional classes that we enhanced by adding both textual definitions and logical definitions connecting to Uberon. An example is 'epithelial cell of thyroid gland' (CL:0002257, FMA:0002257), logically defined as 'endoepithelial cell' and (part of some thymus) [20].

\section{New and revised skeletal cell types}

Work on the Vertebrate Skeletal Anatomy Ontology (VSAO), a unified ontology for the representation of skeletal cells, tissues, biological processes, organs, and subdivisions of the skeletal system [21], resulted in modifications to 13 existing cell types in the CL to ensure that the classes applied across vertebrates, and the addition of 18 new cell types. New relationships between cell types and skeletal tissues were also added, in 
addition to developmental relationships between skeletal cell types. These improvements enable broader queries on skeletal diversity across different biological scales. Improvements in the representation of skeletal tissues, organs, and subdivisions of the skeletal system have since been incorporated from VSAO into the Uberon multi-species anatomy ontology [22], and the logical definitions of associated cells to refer to the Uberon classes.

\section{Extending the $\mathrm{CL}$ to encompass vertebrate diversity}

An ongoing challenge in developing the $\mathrm{CL}$ is to increase the number and granularity of cell types represented for well-studied species such as mouse and human, while providing high level classes needed for the representation of cell types in non-mammalian vertebrates. To ensure that CL classes are applicable to nonmammalian vertebrates two courses of action have been necessary: 1) add non-mammalian classes to the CL; 2) ensure that general cell type definitions do not unintentionally exclude certain organisms. Examples of nonmammalian cell types that have been recently added to the CL include the pigmented cells 'iridoblast' (CL:0005001) and 'xanthoblast' (CL:0005002) [23], and the 'Kolmer-Agduhr neuron' (CL:0005007) [24]. Ensuring that classes are applicable across species is a multifaceted problem and includes optimizing of cell type definitions, as well as (ideally) crafting class hierarchies that incorporate non-mammalian cell types from inception. Cell type definitions can unintentionally exclude non-mammalian vertebrates by including mammalian specific anatomical structures or by including species-specific proteins in the logical definition. At the same time, highly specified cell types for particular taxa are needed to enable querying of complex data using the CL. By adding less specific intermediate classes with inclusive definitions, such as multi-ciliated epithelial cell (CL:0005012), the CL can be used by a wide variety of model organism databases and evolutionary biologists for data annotation, while serving the needs of sophisticated bioinformatics analyses focused on cell types of medical interest.

\section{Improved delineation of content and coordination with other ontologies}

The primary focus of CL is to describe in vivo cell types [3], and while the priority of CL curators has been on in vivo cell types over the past few years, the ontology does in fact include a branch for in vitro cells. In order to clarify the representation of the domain of all cell types, representatives of the $\mathrm{CL}$, Cell Line Ontology (CLO) [25], Reagent Ontology ( $\mathrm{ReO})$ [26], the Gene Ontology [9], and Ontology for Biomedical Investigations (OBI) [27], have agreed that the root class 'cell' (CL:0000000) in CL should be regarded as the root of all cell type classes in OBO Foundry ontologies (Fig. 1), and is equivalent to the GO class 'cell' (GO:0005623). As a result,

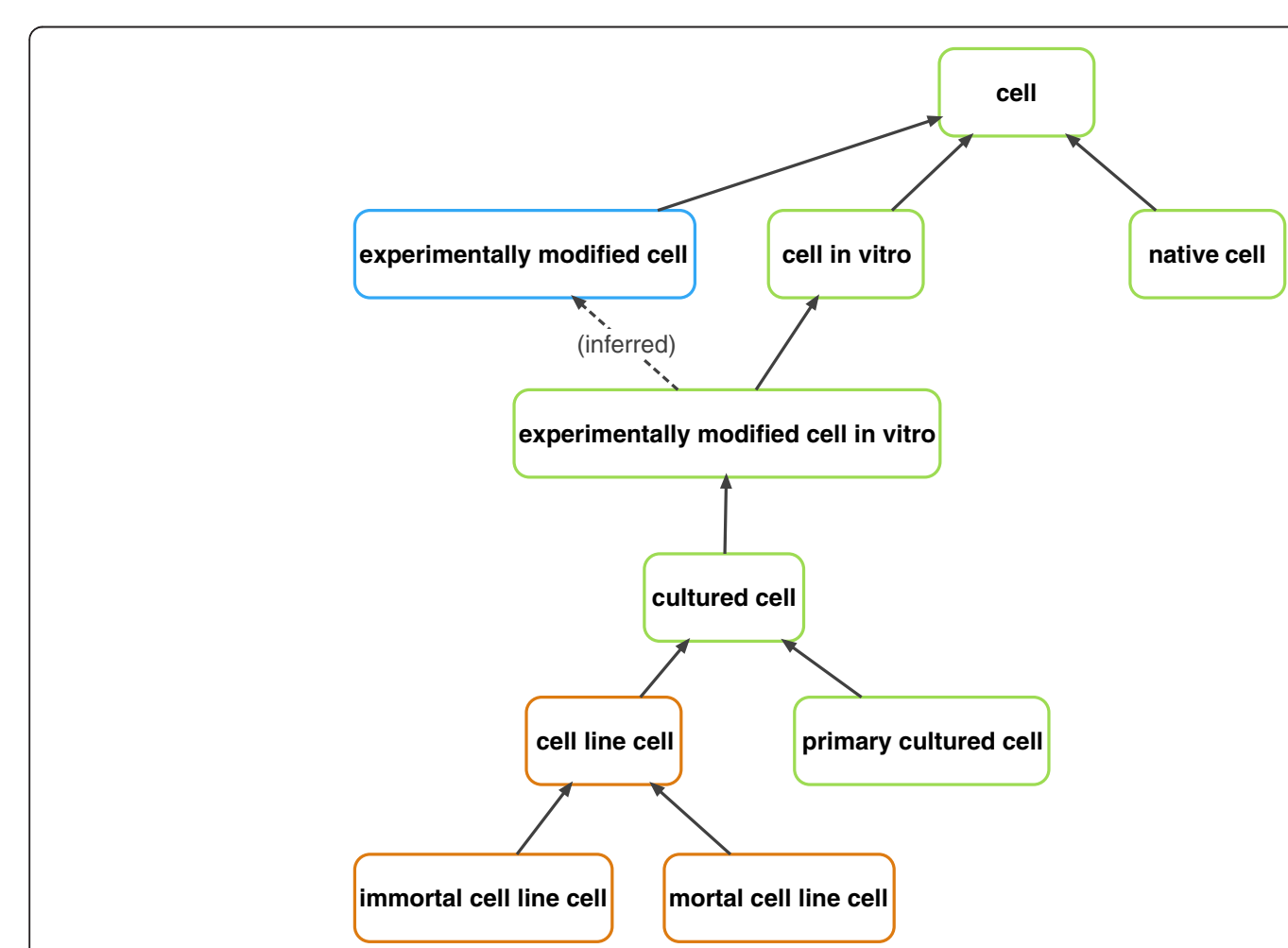

Fig. 1 High level cell types in CL and related ontologies. The hierarchy of high-level cell types is shown. CL nodes: green, ReO: blue, CLO: orange 
changes were made to the upper level classes, to allow for a modular approach that represents in vivo and $e x$ vivo cells types more accurately. Two of the children of the root class 'cell' are 'cell in vitro' (CL:0001034), and 'native cell' (CL:0000003) (which was formerly known as 'cell in vivo'). The definition for 'native cell' reads as follows,

A cell that is found in a natural setting, which includes multicellular organism cells 'in vivo' (i.e. part of an organism), and unicellular organisms 'in environment' (i.e. part of a natural environment).

This definition reflects the fact that while cells of multicellular organisms are naturally considered 'in vivo' in their native state, single celled organisms often inhabit environments that are not part of another organism, and thus are not "in vivo" in that sense. The naturally occurring in vivo cell types of multicellular organisms are therefore properly considered subtypes of 'native cell'.

Another agreed upon change in CL is that the classes 'cell line cell', 'immortal cell line cell', and 'mortal cell line cell' were deprecated (i.e., made obsolete) in CL and replaced with equivalent classes from CLO (see discussion below and Sarntivijai et al. [25] for additional details). As CLO specifically represents cell line cells, it seemed appropriate for CLO to contain its own root class and high-level cell type classes, and for the CLO developers to assume editorial control for these classes. Where needed, these three CLO classes were imported into CL using the MIREOT method $[28,29]$ to support existing annotations to these classes, and users of these classes, primarily MGI [16], were informed well in advance of these changes.

Similarly, $\mathrm{ReO}[26]$ contains the class 'experimentally modified cell' (Fig. 1) and a variety of related classes such as 'genetically modified cell' and 'experimentally modified multicellular organism cell in vivo'. These cell type classes most commonly denote reagents of some type and fall outside of the domain of the CL proper, and clearly are within the domain of $\mathrm{ReO}$.

Plant cell types and insect cell types are now handled independently of the $\mathrm{CL}$ as separate modules. The Plant Ontology (PO) has recently undergone new developments and the PO team has taken responsibility for curation of all plant cell type classes [30]. Consequently, all plant cell type classes in CL have been made obsolete. These plant cell types classes in the CL were already duplicates of existing PO classes, and were thus redundant and confusing to users. PO cell type classes may be imported into an extended version of $\mathrm{CL}$ as an OWL import in the future, retaining their PO IDs. [31]. A similar process is already used to create a pan-metazoan version of $\mathrm{CL}$ as part of the
Uberon release process [32]; this will be extended to include Viridiplantae.

While the CL continues to represent a number of high level insect cell types, the Drosophila Anatomy Ontology (FBbt) contains cell types for many insect cell types not represented in CL, particularly insect neurons [33-35]. Similarly, the Zebrafish Anatomy Ontology (ZFA) also contains neuron types not represented in CL [36]. Going forward, the general approach is that non-mammalian species-specific cell types will be represented as is_a children of the appropriate $\mathrm{CL}$ parent in the speciesspecific anatomy ontology when such an ontology exists. The CL will continue to maintain general cell types for representation of non-mammalian cells where no separate resource or ontology exists and will remain the principal ontology for the representation of mammalian cell types.

As described above, the root class 'cell' (CL:0000000) in $\mathrm{CL}$ is declared to be logically equivalent to the $\mathrm{GO}$ class 'cell' (GO:0005623), within the Cell Ontology. While this arrangement mostly works for practical use of the $\mathrm{CL}$, a long class proposal has been to deprecate 'cell' (CL:0000000) and simply make the GO class 'cell' (GO:0005623) to be the root of the Cell Ontology. However, there are still some minor differences in the way the two classes are defined, and questions about whether the Gene Ontology with its orientation to describing 'normal' or physiological biology should provide the CL root node 'cell', whose subtypes include tumor cell types, cell line cell types, and other experimentally modified cell types. This issue awaits additional discussion with the Gene Ontology Consortium and other interested parties.

\section{Natural Language and Logical Definitions in CL}

The proportion of classes with natural language definitions has remained relatively constant, with a coverage of $82 \%$ in both 2011 and the present. We still aim to boost this proportion to have $100 \%$ coverage. The last five years have seen general improvements in logical axiomatization-in 2011 we reported the number of classes with defining equivalence axioms (logical definitions) to be 340 , this number has increased to 1534, added through both manual and automated methods $[3,20]$.

The set of ontologies imported into the CL to provide logical definitions remains constant, and consists of Uberon [22, 37], Protein Ontology (PRO) [38], GO [9], the Chemical Entities of Biological Interest (ChEBI) ontology [39], and the Phenotypic And Trait Ontology (PATO) [40]. Some classes make use of a variety of classes in the same axiom, such as 'T-helper 1 cell', which includes a mix of relations to both PRO classes and GO classes (Fig. 2). 


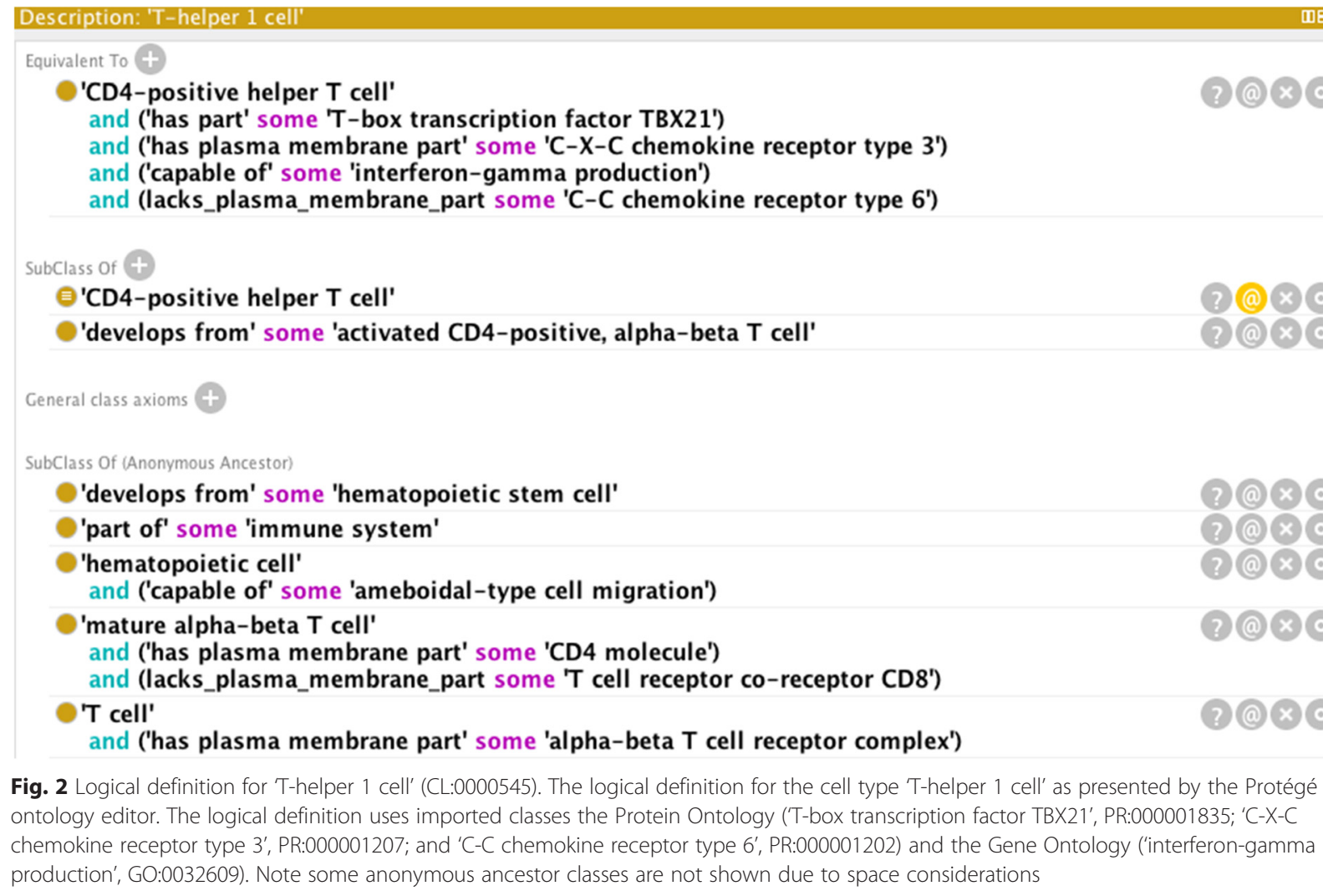

Fig. 2 Logical definition for 'T-helper 1 cell' (CL:0000545). The logical definition for the cell type 'T-helper 1 cell' as presented by the Protégé ontology editor. The logical definition uses imported classes the Protein Ontology ('T-box transcription factor TBX21', PR:000001835; 'C-X-C chemokine receptor type 3', PR:000001207; and 'C-C chemokine receptor type 6', PR:000001202) and the Gene Ontology ('interferon-gamma production', GO:0032609). Note some anonymous ancestor classes are not shown due to space considerations

\section{Improvements to nervous system cell types}

In order to improve the representation of neurons and related cell types, we adopted the relations and methods originally developed from the Drosophila Anatomy Ontology $[34,35]$. These include synapsed_to and has_synaptic_terminal_in, used to capture connectivity of neurons to each other and larger anatomical structures. We aim to coalesce with other neuron-specific vocabularies and ontologies, in particularly those that were part of the Neuroscience Information Framework (NIF) Standard suite of ontologies [41]. The analogous task has already been performed for neuron parts [42], and the gross neuroanatomical structure subset of NIFSTD has been incorporated into Uberon. As an initial task, we have aligned the contents of NIF-Cell with the CL by matching up identical or similar classes in the two hierarchies to identify gaps in both ontologies and differences in the ontologies' structures. We will then define standard patterns for neuronal cell types, and import missing neuron cell types from NIF. In order to synchronize with the corresponding Neurolex wiki system, we have developed an approach for translating the Neurolex semantic wiki into OWL [43].

Recent improvements in CL development methodology The CL was originally developed using the OBO-Format and the OBO-Edit ontology editor [1, 44], without any automated quality control, release pipeline or automated procedures for building the ontology. We previously reported on improvements to this methodology, specifically leveraging the OWL2 ontology language [45] and associated tooling such as OWL reasoners, and the Protégé $5 . x$ editor $[20,46]$.

We have made further changes and improvements to the ontology engineering framework we use. Previously, the editors' version (source code) for the ontology was in OBO-Format, necessitating a conversion to OWL step prior to reasoning and debugging in Protégé. We have since switched the editors' version to be in OWL, simplifying the procedure for working with the OWL stack of tools (note that we still produce editions in OBOFormat along with every release, as many bioinformatics tools still rely on this format). This switch also gives us greater flexibility for expressing concepts using the richer constructs available in the OWL language.

We have also implemented a TermGenie [47] instance, available at cl.termgenie.org. This provides a wider community of users a web frontend for instant provisioning of new classes, either conforming to pre-defined templates (i.e. design patterns), or templateless free-form additions. Currently the only design pattern implemented is a simple 'part-whole' template for the addition of classes like 'epithelial cell of forearm'. One of the main users 
of the TermGenie instance has been the curators of the ENCODE project (see below).

We make use of the Jenkins Continuous Integration system, as developed and implemented by the Gene Ontology Consortium, for quality control and validation $[8,48]$. This system alerts the editorial team if changes are made that inadvertently introduce logical, terminological, or structural errors into the ontology (for example, a cell that is located in two disconnected locations, or two cell classes that share the same name). We are in the process of switching to Travis-CI as this provides more direct integration with the GitHub system, where we manage the ontology. This system is also used to generate releases, creating a package of ontology files in OWL2 and OBO formats that are pre-reasoned and in some cases simplified for legacy use for systems that do not support logical definitions (See Table 1 for listing of available CL files).

In the time since we last published on CL, we have migrated the source repository we use to manage the ontology on two occasions. We originally migrated from SourceForge to GoogleCode; some time later, Google announced the retirement of GoogleCode, so we then followed many other ontologies and migrated to GitHub, where the source now resides [49]. Note however that most users of the CL do not interact with GitHub directly, and retrieve the ontology from the URLs provided in Table 1. Class requests and other inquiries for the ontology developers should be made through the CL issue tracker [50]. We have deprecated the older issue trackers on SourceForge and GoogleCode, and we migrated the tickets on these systems to GitHub.

While this migration process caused some disruption, this is compensated by efficiencies afforded by the GitHub system-for example, the ability to link edits on the ontology to tickets. The GitHub release mechanism also works well for ontology releases. One feature we hope to deploy this year is the ability to move to a GitHub-flow style of development, allowing external editors the ability to make 'pull requests' on the ontology, with complete validation being performed by Travis.

\begin{tabular}{|c|c|c|c|}
\hline File & $\begin{array}{l}\text { pre- } \\
\text { reasoned }\end{array}$ & $\begin{array}{l}\text { with external } \\
\text { ontology classes }\end{array}$ & PURL \\
\hline cl-edit.owl & no & yes & $\mathrm{N} / \mathrm{A}$ \\
\hline cl.owl & yes & yes & $\begin{array}{l}\text { http://purl.obolibrary.org/obo/ } \\
\text { cl.owl }\end{array}$ \\
\hline cl.obo & yes & yes & $\begin{array}{l}\text { http://purl.obolibrary.org/obo/ } \\
\text { cl.obo }\end{array}$ \\
\hline cl-basic.owl & yes & no & $\begin{array}{l}\text { http://purl.obolibrary.org/obo/ } \\
\text { cl-basic.owl }\end{array}$ \\
\hline cl-basic.obo & yes & no & $\begin{array}{l}\text { http://purl.obolibrary.org/obo/ } \\
\text { cl-basic.obo }\end{array}$ \\
\hline
\end{tabular}

\section{Utility and discussion}

Use of CL classes in development of other ontologies

Cells are central to understanding biology from the molecular to the organismal level, and the CL is increasingly useful as a tool for representing and organizing cell types and data related to cell types in a variety of projects. As the designated ontology for the representation of cells in the OBO Foundry, the CL is used in a number of ontologies for the development of compositional classes via logical definitions. Gene Ontology developers have long employed the principle of "cross-product" class development, in which two classes from different ontologies are combined to make a more expressive "pre-composed" (or "compositional") ontology class [6-8]. The class 'neuron differentiation' (GO:0030182), for instance has the logical axiom 'cell differentiation' and (results in acquisition of features of some 'neuron'), where 'neuron' is a CL class. As GO developers continue to implement logical definitions for crossproduct classes, they have increasingly needed new cell types in CL for use in these logical definitions. In order to facilitate this process, GO ontology developers have been trained in CL ontology editing as well and are now making direct contributions to the CL. An extended version of the $\mathrm{GO}$ that includes a subset of the $\mathrm{CL}$ together with linking axioms is available [51].

Development of the Cell Line Ontology (CLO) has referenced CL cell types and the hierarchy of the CL [25]. In CLO, all cell line cells are under the CLO class 'cell line cell', which is a child of CL 'cultured cell'. Initially, CLO listed over 30,000 cell line cells immediately under the parent class 'cell line cell'. To better identify the relations among different cell line cells, CLO generated many intermediate cell line cell classes (e.g., 'immortal epidermal cell line cell' and 'immortal keratinocyte cell line cell') based on a basic relation design that a CLO 'cell line cell' is derived from a CL 'cell', for example, CLO 'immortal epidermal cell line cell' derives from some 'epidermal cell', and CLO 'immortal keratinocyte cell line cell' derives_from some 'keratinocyte'. In CL, the class 'epidermal cell' is a parent of 'keratinocyte'. Based on this CL hierarchical definition, CLO automatically includes a logical definition that immortal epidermal cell line cell' is a parent of 'immortal keratinocyte cell line cell'. In total over $130 \mathrm{CL}$ classes were imported to CLO with the hierarchy of the CL informing CLO structure. These newly generated CL-matched CLO classes were then used as parent classes for the over 30,000 cell line cells in CLO to layout an improved hierarchy of the cell line cells [25].

Interactions between different ontologies in the scope of biological cells can become complicated as we implement a thorough and precise representation of knowledge in this domain. As described above, CLO's 'cell line cell' is a subclass of CL's 'experimentally modified 
cell in vitro' where 'experimentally modified cell in vitro' is inferred as a subclass of ReO's 'experimentally modified cell'. The correct relationships among these related classes are only seen when all have been loaded into Protégé and a reasoner has been run. This degree of interrelatedness and complexity is becoming more common in bio-ontology practice, and demonstrates the needs for effective communication within the community. Being the center of interactions in situation like this, the CL has acted as the facilitating moderator of this kind of communication.

CL development allows for modular development of species-specific extensions. These extensions enable the creation of very granular cell types defined in ways that are unique to a particular species or limited to a subset of species. However, many cell types can be generically defined across species, and the CL provides the appropriate $\mathrm{OBO}$ ontology for their representation. In order to allow for comparison and integration of cell type specific data between species, species-specific cell types should always be subtypes of generic CL cell types. While development of modular extensions to CL is encouraged, the well-developed hierarchy of classes in the CL provides a valuable resource for data annotators working in species who do not have time or resources to develop CL extensions.

As examples of this methodology, developers of species-specific anatomy ontologies such as the Zebrafish Anatomical Ontology (ZFA) [36] and the Xenopus Anatomy Ontology (XAO) [52] have extended the CL by incorporating species-specific cell classes as is_a children of CL classes in their ontologies. This strategy allows ontologists to make species-specific classes that are is_a children of the appropriate CL class for use in data annotation at model organism databases. The integration of the CL with the species-specific ontologies also allows the CL classes to be used in phenotype and expression annotations at ZFIN [15] and expression annotations at Xenbase [53].

As ontologies such as the Infectious Disease Ontology (IDO) [54] or the Neurological Disease Ontology [55] are developed, CL classes are being used to represent information such as viral tropism or neurons affected in Parkinson's disease. As with the GO, there is communication between developers of related biomedical ontologies that contribute to the development of both. The $\mathrm{CL}$ is also a component of the Experimental Factor Ontology (EFO), used to provide descriptions of experimental variables in databases at the European Bioinformatics Institute [56].

The $\mathrm{CL}$ is also being used far more extensively in the $\mathrm{GO}$, in particular the GO has added a way to provide additional cellular context to gene associations using a mechanism called "annotation extensions" [57]. These cross-ontology linkages are used by a number of model organism databases in GO annotation and visible in AmiGO-for example, the page for 'neuron' includes GO annotations for neuronal parts [58].

\section{Use of the $\mathrm{CL}$ as Metadata in ENCODE and FANTOM5 Projects}

Two major projects studying gene expression have utilized the CL as part of their data analysis pipelines. The Encyclopedia of DNA Elements (ENCODE) Consortium, which is funded and organized by National Human Genome Research Institute (NHGRI), aims to discover and define the functional elements encoded in the human genome [59]. ENCODE investigators are utilizing a prioritized set of various cell types to complete annotations about genes and their RNA transcripts and transcriptional regulatory regions and have developed data standards that utilizes the $\mathrm{CL}$, among other ontologies, to describe the metadata for cell types used and experimental assays $[60,61]$.

The value of the CL for data integration and analyses was adeptly demonstrated in a recent series of notable papers from the FANTOM5 Consortium, which relied in part on the CL for large-scale data analyses of transcriptional start sites [62], enhancers [63], and waves of transcription in differentiating cell types [64]. The FANTOM5 Consortium utilized the $\mathrm{CL}$ as a component of the FANTOM Sample Ontology, in combination with Uberon, the Disease Ontology and the EFO to identify cellular, tissue, disease sources and experimental modifications for the samples used in transcriptional analyses [65]. By relying on the ontological hierarchy provided by the CL, the FANTOM5 Consortium was able to classify transcription patterns associated with individual cell types, groupings of related cell types, and cell lineages during differentiation $[62,64]$.

\section{Use of the $\mathrm{CL}$ in other non-ontology projects}

The CL is being used as metadata in a variety of nonontology projects, such as The Cell: An Image Library [66], CELLPEDIA [67], Phenoscape [13], LINCS [68], the Human Immunology Project Consortium (HIPC) [11], and ImmPort [10]. The HIPC and ImmPort projects are National Institute of Allergy and Infectious Diseases (NIAID) sponsored programs to collect and organize data from immunology experiments performed by NIAID supported investigators in order to facilitate secondary usage [10]. In support of these projects, the $\mathrm{CL}$ is being used both as a controlled vocabulary of cell types for use as metadata, and as part of an analytical pipeline for analyzing high-dimensional flow cytometry and mass cytometry data (e.g. CyTOF) [69] submitted to the ImmPort data repository. Developers of the CL have already incorporated novel B cell types discovered via 
high-dimensional flow cytometry [70], such as 'IgG-positive double negative memory B cell' (CL:0002103) and 'IgD-negative CD38-positive IgG memory B cell' (CL:0002107). The CyTOF method is yielding information about even more granular cell types [71]. In order to facilitate the analysis of data generated in highdimensional flow cytometry or CyTOF, the flowCL software package matches cell populations identified via automated gating algorithms against existing cell types in the CL based on their combinations of markers, or immunophenotypes [72, 73].

\section{Conclusion}

Through cooperative efforts between the Cell Ontology editors and various stakeholders, ongoing development of the CL has ensured that it continues to be a valuable resource for users and developers of related ontologies. Use of the CL by a broad range of third party efforts, including the high visibility ENCODE and FANTOM5 projects, as a source of metadata and for data integration and analysis shows the value of the CL to the wider scientific community. As big data collection and analysis continues to grow in importance as a source of biological discovery, we expect the CL will be of key utility in organizing and understanding these data. We invite community feedback and participation to continue the improvements to the CL.

\section{Availability and requirements}

Like all OBO library ontologies, the $\mathrm{CL}$ is available from a standard purl [http://purl.obolibrary.org/obo/cl.owl]. The main URL for the project

[http://cellontology.org/] and links to various browsers are available from the main OBO Library page for CL [http://obofoundry.org/ontology/cl.html].

\footnotetext{
Acknowledgements

We would kindly thank Barry Smith, Lindsay Cowell, Anna Maria Masci, Richard Scheuermann, Jose Mejino, David Hill, Terry Hayamizu, Morgan Hightshoe, Wade Valleau, Jane Lomax, Paola Roncaglia, Tanya Berardini, Heiko Dietze, Maryann Martone, Stephan Larson, Gordon Shepherd, Jyl Boline, Mihail Bota, Giorgio Ascoli, Paul Katz, Robert Burgess, Patrick Ray, Jonathan Bona, Paula Mabee, Laurel Cooper, Ramona Walls, Pankaj Jaiswal, Darren Natale, Cathy Wu, Cecilia Arighi, Alistair Forrest, Hideya Kawaji, Helen Parkison, Simon Jupp, Robert Stevens, Ryan Brinkmann, Melanie Courtot, Raphael Gottardo, Cliburn Chan, Jie Zheng, Shai Shen-Orr, and Yannick Pouliot, for discussions about and contributions to the Cell Ontology project. ADD, TFM, CJM, and JAB were supported by NHGRI grants HG002273-09Z and HG002273 for portions of this work. CJM's work was supported by the Director, Office of Science, Office of Basic Energy Sciences, of the U.S. Department of Energy under Contract No. DE-AC02-05CH11231. ADD and AR are supported by NIGMS grant 2R01GM080646-06 and NIAID contract HHSN272201200028C for portions of this work. YMB and CVS are supported by $\mathrm{NIH} \mathrm{HG002659}$ for portions of this work. MAH, MHB, and NAV are supported for portion of this work by 1R24OD011883 from the NIH Office of the Director. WD is supported by NSF grants DBI-0641025, DBI-1062404, and DBI-1062542, and by the National Evolutionary Synthesis Center under NSF EF-0423641 and NSF EF-0905606 for portions of this work. YH was supported by NIH 1R01Al081062. Any opinions, findings, and conclusions or recommendations expressed in this material are those of the author(s) and do not necessarily reflect the views of the
}

National Science Foundation or the National Institutes of Health. We gratefully acknowledge the support of the International Neuroinformatics Coordinating Facility for portions of this work.

\section{Authors' contributions}

All authors have contributed to CL ontology development through discussions of key issues and/or contributions of ontology classes. CJM developed the $C L$ production system. The $C L$ project is managed by ADD, $A R, M A H, J A B$, and $C J M$. ADD wrote the manuscript with contributions from YMB, WMD, YH, CVS, DOS, MAH, NV, SS, TFM, and CJM. All authors read and approved the manuscript.

\section{Competing interests}

The authors declare that they have no competing interests.

\section{Author details}

'Department of Neurology, University at Buffalo School of Medicine and Biomedical Sciences, Buffalo, NY 14203, USA. ${ }^{2}$ European Molecular Biology Laboratory, European Bioinformatics Institute, Hinxton, Cambridge CB10 1SD, UK. ${ }^{3}$ ZFIN, the Zebrafish Model Organism Database, 5291 University of Oregon, Eugene, OR 97403, USA. ${ }^{4}$ Ontology Development Group, Library, Oregon Health and Science University, Portland, Oregon 97239, USA. ${ }^{5}$ Department of Biology, University of South Dakota, Vermillion, SD 57069, USA. ${ }^{6}$ National Evolutionary Synthesis Center, Durham, NC 27705, USA. ${ }^{7}$ Southwestern Medical Center, University of Texas, Dallas, TX 75235, USA. ${ }^{8}$ Unit for Laboratory Animal Medicine, University of Michigan Medical School, Ann Arbor, Ml 48109, USA. ${ }^{9}$ Oral Diagnostics Sciences, University at Buffalo School of Dental Medicine, Buffalo, NY 14210, USA. ${ }^{10}$ The Jackson Laboratory, Bar Harbor, ME 04609, USA. ${ }^{11}$ Genomics Division, Lawrence Berkeley National Laboratory, Berkeley, CA 94720, USA.

Received: 15 April 2016 Accepted: 23 June 2016

Published online: 04 July 2016

\section{References}

1. Bard J, Rhee SY, Ashburner M. An ontology for cell types. Genome biology. 2005;6:R21.

2. Diehl AD, Augustine AD, Blake JA, Cowell LG, Gold ES, Gondre-Lewis TA, Masci AM, Meehan TF, Morel PA, Nijnik A, et al. Hematopoietic cell types: prototype for a revised cell ontology. J Biomed Informat. 2011:44:75-9.

3. Meehan TF, Masci AM, Abdulla A, Cowell LG, Blake JA, Mungall CJ, Diehl AD. Logical development of the cell ontology. BMC Bioinformatics. 2011;12:6.

4. Masci AM, Arighi CN, Diehl AD, Lieberman AE, Mungall C, Scheuermann RH, Smith B, Cowell LG. An improved ontological representation of dendritic cells as a paradigm for all cell types. BMC Bioinformatics. 2009;10:70.

5. Smith B, Ashburner M, Rosse C, Bard J, Bug W, Ceusters W, Goldberg L, Eilbeck K, Ireland A, Mungall CJ, et al. The OBO Foundry: coordinated evolution of ontologies to support biomedical data integration. Nature Biotechnol. 2007:25:1251-5.

6. Hill DP, Blake JA, Richardson JE, Ringwald M. Extension and integration of the gene ontology $(\mathrm{GO})$ : combining $\mathrm{GO}$ vocabularies with external vocabularies. Genome Res. 2002;12:1982-91.

7. Mungall CJ, Bada M, Berardini TZ, Deegan J, Ireland A, Harris MA, Hill DP, Lomax J. Cross-product extensions of the Gene Ontology. J Biomed Informat. 2011:44:80-6.

8. Gene Ontology C. Gene Ontology Consortium: going forward. Nucleic Acids Res. 2015:43:D1049-1056.

9. Ashburner M, Ball CA, Blake JA, Botstein D, Butler H, Cherry JM, Davis AP, Dolinski K, Dwight SS, Eppig JT, et al. Gene ontology: tool for the unification of biology. The Gene Ontology Consortium. Nat Genet. 2000;25:25-9.

10. Bhattacharya S, Andorf S, Gomes L, Dunn P, Schaefer H, Pontius J, Berger P, Desborough V, Smith T, Campbell J, et al. ImmPort: disseminating data to the public for the future of immunology. Immunol Res. 2014;58:234-9.

11. Maecker HT, McCoy JP, Nussenblatt R. Standardizing immunophenotyping for the Human Immunology Project. Nat Rev Immunol. 2012;12:191-200.

12. Dahdul WM, Balhoff JP, Engeman J, Grande T, Hilton EJ, Kothari C, Lapp H, Lundberg JG, Midford PE, Vision TJ, et al. Evolutionary characters, phenotypes and ontologies: curating data from the systematic biology literature. PloS One. 2010;5:e10708. 
13. Mabee BP, Balhoff JP, Dahdul WM, Lapp H, Midford PE, Vision TJ, Westerfield M. 500,000 fish phenotypes: The new informatics landscape for evolutionary and developmental biology of the vertebrate skeleton. Zeitschrift fur angewandte Ichthyologie $=J$ Appl Ichthyol. 2012;28:300-5.

14. Mungall CJ, Washington NL, Nguyen-Xuan J, Condit C, Smedley D, Kohler S, Groza T, Shefchek K, Hochheiser H, Robinson PN, et al. Use of model organism and disease databases to support matchmaking for human disease gene discovery. Hum Mutat. 2015;36:979-84.

15. Howe DG, Bradford YM, Conlin T, Eagle AE, Fashena D, Frazer K, Knight J, Mani P, Martin R, Moxon SA, et al. ZFIN, the Zebrafish Model Organism Database: increased support for mutants and transgenics. Nucleic Acids Res. 2013;41:D854-860

16. Bult CJ, Eppig JT, Blake JA, Kadin JA, Richardson JE, Mouse Genome Database G. Mouse genome database 2016. Nucleic Acids Res. 2016;44: D840-847.

17. Jupp S, Klein J, Schanstra J, Stevens R. Developing a kidney and urinary pathway knowledge base. J Biomed Semant. 2011;2 Suppl 2:S7.

18. Alam-Faruque Y, Hill DP, Dimmer EC, Harris MA, Foulger RE, Tweedie S, Attrill H, Howe DG, Thomas SR, Davidson D, et al. Representing kidney development using the gene ontology. PloS One. 2014;9:e99864.

19. Rosse C, Mejino Jr JL. A reference ontology for biomedical informatics: the Foundational Model of Anatomy. J Biomed Informat. 2003;36:478-500

20. Meehan TF, Mungall CJ, Diehl AD. Revising the Cell Ontology. Buffalo: Proceedings of the 2nd International Conference on Biomedical Ontology; 2011. http://ceur-ws.org/Vol-833/paper2.pdf. Accessed 28 June 2016

21. Dahdul WM, Balhoff JP, Blackburn DC, Diehl AD, Haendel MA, Hall BK, Lapp $H$, Lundberg JG, Mungall CJ, Ringwald $M$, et al. A unified anatomy ontology of the vertebrate skeletal system. PloS One. 2012;7:e51070.

22. Haendel MA, Balhoff JP, Bastian FB, Blackburn DC, Blake JA, Bradford Y, Comte A, Dahdul WM, Dececchi TA, Druzinsky RE, et al. Unification of multispecies vertebrate anatomy ontologies for comparative biology in Uberon. J Biomed Semant. 2014;5:21.

23. Curran K, Lister JA, Kunkel GR, Prendergast A, Parichy DM, Raible DW. Interplay between Foxd3 and Mitf regulates cell fate plasticity in the zebrafish neural crest. Dev Biol. 2010;344:107-18.

24. Dale N, Roberts A, Ottersen OP, Storm-Mathisen J. The morphology and distribution of 'Kolmer-Agduhr cells', a class of cerebrospinal-fluidcontacting neurons revealed in the frog embryo spinal cord by GABA immunocytochemistry. Proceedings of the Royal Society of London Series B, Biological Sciences. 1987;232:193-203.

25. Sarntivijai S, Lin Y, Xiang Z, Meehan TF, Diehl AD, Vempati UD, Schurer SC, Pang C, Malone J, Parkinson H, et al. CLO: The cell line ontology. J Biomed Semant. 2014;5:37.

26. Brush MH, Vasilevsky N, Torniai C, Johnson T, Shaffer C, Haendel MA Developing A Reagent Application Ontology Within The OBO Foundry Framework. Buffalo: Proceedings of the 2nd International Conference on Biomedical Ontology; 2011. http://ceur-ws.org/Nol-833/paper32.pdf. Accessed 28 June 2016.

27. Brinkman RR, Courtot M, Derom D, Fostel JM, He Y, Lord P, Malone J, Parkinson H, Peters B, Rocca-Serra P, et al. Modeling biomedical experimental processes with OBI. J Biomed Semant. 2010;1 Suppl 1:S7.

28. Courtot M, Gibson F, Lister AL, Malone J, Schober D, Brinkman RR Ruttenberg A. MIREOT: The minimum information to reference an external ontology term. Applied Ontology. 2011;6:23-33.

29. Xiang Z, Courtot M, Brinkman RR, Ruttenberg A, He Y. OntoFox: web-based support for ontology reuse. BMC Res Notes. 2010;3:175.

30. Cooper L, Walls RL, Elser J, Gandolfo MA, Stevenson DW, Smith B, Preece J, Athreya B, Mungall CJ, Rensing S. The plant ontology as a tool for comparative plant anatomy and genomic analyses. Plant Cell Physiol. 2013;54:e1.

31. Mungall CJ, Haendel M, Ireland A, Manzoor S, Meehan T, Osumi-Sutherland D, Torniai C, Diehl AD. Modularization for the Cell Ontology. In: Proceedings of ICBO: International Conference on Biomedical Ontology. 2011. p. 370-376

32. Composite (merged) Multispecies Ontologies [http://uberon.github.io/ downloads.html\#multiont] Accessed 15 June 2016.

33. Milyaev N, Osumi-Sutherland D, Reeve S, Burton N, Baldock RA, Armstrong JD. The Virtual Fly Brain browser and query interface. Bioinformatics. 2012;28:411-5
34. Osumi-Sutherland D, Reeve S, Mungall CJ, Neuhaus F, Ruttenberg A, Jefferis GS, Armstrong JD. A strategy for building neuroanatomy ontologies. Bioinformatics. 2012;28:1262-9.

35. Costa M, Reeve S, Grumbling G, Osumi-Sutherland D. The Drosophila anatomy ontology. J Biomed Semant. 2013;4:32.

36. Van Slyke CE, Bradford YM, Westerfield M, Haendel MA. The zebrafish anatomy and stage ontologies: representing the anatomy and development of Danio rerio. J Biomed Semant. 2014;5:12.

37. Mungall CJ, Torniai C, Gkoutos GV, Lewis SE, Haendel MA. Uberon, an integrative multi-species anatomy ontology. Genome Biol. 2012:13:R5.

38. Natale DA, Arighi CN, Blake JA, Bult CJ, Christie KR, Cowart J, D'Eustachio P, Diehl AD, Drabkin HJ, Helfer O, et al. Protein Ontology: a controlled structured network of protein entities. Nucleic Acids Res. 2014;42:D415-421.

39. Degtyarenko K, de Matos P, Ennis M, Hastings J, Zbinden M, McNaught A, Alcantara R, Darsow M, Guedj M, Ashburner M. ChEBI: a database and ontology for chemical entities of biological interest. Nucleic Acids Res. 2008; 36:D344-350.

40. Gkoutos GV, Mungall C, Dolken S, Ashburner M, Lewis S, Hancock J, Schofield P, Kohler S, Robinson PN. Entity/quality-based logical definitions for the human skeletal phenome using PATO. Conference proceedings : Annual International Conference of the IEEE Engineering in Medicine and Biology Society IEEE Engineering in Medicine and Biology Society Conference. 2009:2009:7069-72.

41. Bug WJ, Ascoli GA, Grethe JS, Gupta A, Fennema-Notestine C, Laird AR, Larson SD, Rubin D, Shepherd GM, Turner JA, Martone ME. The NIFSTD and BIRNLex vocabularies: building comprehensive ontologies for neuroscience. Neuroinformatics. 2008:6:175-94

42. Roncaglia P, Martone ME, Hill DP, Berardini TZ, Foulger RE, Imam FT, Drabkin H, Mungall CJ, Lomax J. The Gene Ontology (GO) Cellular Component Ontology: integration with SAO (Subcellular Anatomy Ontology) and other recent developments. J Biomed Semant. 2013;4:20.

43. Prolog utilities for querying and extracting information from NeuroLex [https://github.com/cmungall/nlx-pl]

44. Day-Richter J, Harris MA, Haendel M, Gene Ontology OBOEWG, Lewis S. OBO-Edit-an ontology editor for biologists. Bioinformatics. 2007;23: 2198-200.

45. Hitzler P, Krötzsch M, Parsia B, Patel-Schneider PF, Rudolph S. OWL 2 Web Ontology Language Primer (Second Edition), W3C; 2012. http://www.w3. org/TR/owl2-primer. Accessed 28 June 2016

46. Noy N, Tudorache T, Nyulas C, Musen M. The ontology life cycle: Integrated tools for editing, publishing, peer review, and evolution of ontologies. AMIA Annual Symposium proceedings / AMIA Symposium AMIA Symposium. 2010;2010:552-6.

47. Dietze H, Berardini TZ, Foulger RE, Hill DP, Lomax J, Osumi-Sutherland D, Roncaglia P, Mungall CJ. TermGenie - a web-application for pattern-based ontology class generation. J Biomed Semant. 2014;5:48.

48. Continuous Integration of Open Biological Ontology Libraries [http://bioontologies.knowledgeblog.org/405] Accessed 15 June 2016.

49. Cell Ontology Github Page [https://github.com/obophenotype/cellontology] Accessed 15 June 2016.

50. Cell Ontology Issue Tracker [http://purl.obolibrary.org/obo/cl/tracker] Accessed 15 June 2016.

51. go-plus.owl [http://purl.obolibrary.org/obo/go/extensions/go-plus.owl] Accessed 15 June 2016.

52. Segerdell E, Bowes JB, Pollet N, Vize PD. An ontology for Xenopus anatomy and development. BMC Dev Biol. 2008:8:92.

53. Karpinka JB, Fortriede JD, Burns KA, James-Zorn C, Ponferrada VG, Lee J, Karimi K, Zorn AM, Vize PD. Xenbase, the Xenopus model organism database; new virtualized system, data types and genomes. Nucleic Acids Res. 2015:43:D756-763.

54. Cowell LG, Smith B. Infectious disease ontology. In: Infectious disease informatics. New York, USA: Springer-Verlag; 2010. p. 373-395

55. Jensen M, Cox AP, Chaudhry N, Ng M, Sule D, Duncan W, Ray P, WeinstockGuttman B, Smith B, Ruttenberg A, et al. The neurological disease ontology. J Biomed Semant. 2013:4:42.

56. Malone J, Holloway E, Adamusiak T, Kapushesky M, Zheng J, Kolesnikov N, Zhukova A, Brazma A, Parkinson H. Modeling sample variables with an Experimental Factor Ontology. Bioinformatics. 2010;26:1112-8.

57. Huntley RP, Harris MA, Alam-Faruque Y, Blake JA, Carbon S, Dietze H, Dimmer EC, Foulger RE, Hill DP, Khodiyar VK. A method for increasing 
expressivity of Gene Ontology annotations using a compositional approach. BMC Bioinformatics. 2014;15:155.

58. AmiGO page for 'neuron' [http://amigo.geneontology.org/amigo/term/CL: 0000540] Accessed 15 June 2016.

59. Encode Project C, Dunham I, Kundaje A, Aldred SF, Collins PJ, Davis CA, Doyle F, Epstein CB, Frietze S, Harrow J, et al. An integrated encyclopedia of DNA elements in the human genome. Nature. 2012;489:57-74.

60. Malladi VS, Erickson DT, Podduturi NR, Rowe LD, Chan ET, Davidson JM, Hitz BC, Ho M, Lee BT, Miyasato S, et al. Ontology application and use at the ENCODE DCC. Database: J Biol Databases and Curation. 2015;2015:bav010.

61. Sloan CA, Chan ET, Davidson JM, Malladi VS, Strattan JS, Hitz BC, Gabdank I, Narayanan AK, Ho M, Lee BT, et al. ENCODE data at the ENCODE portal. Nucleic Acids Res. 2016;44:D726-732.

62. FANTOM Consortium and the RIKEN PMI and CLST (DGT), Forrest AR, Kawaji H, Rehli M, Baillie JK, de Hoon MJ, Haberle V, Lassmann T, Kulakovskiy IV, Lizio M, et al. A promoter-level mammalian expression atlas. Nature. 2014; 507:462-70.

63. Andersson R, Gebhard C, Miguel-Escalada I, Hoof I, Bornholdt J, Boyd M, Chen Y, Zhao X, Schmidl C, Suzuki T, et al. An atlas of active enhancers across human cell types and tissues. Nature. 2014;507:455-61.

64. Arner E, Daub CO, Vitting-Seerup K, Andersson R, Lilje B, Drablos F, Lennartsson A, Ronnerblad M, Hrydziuszko O, Vitezic M, et al. Transcribed enhancers lead waves of coordinated transcription in transitioning mammalian cells. Science. 2015:347:1010-4.

65. Lizio M, Harshbarger J, Shimoji H, Severin J, Kasukawa T, Sahin S, Abugessaisa I, Fukuda S, Hori F, Ishikawa-Kato S, et al. Gateways to the FANTOM5 promoter level mammalian expression atlas. Genome Biol. 2015 16:22.

66. Orloff DN, Iwasa JH, Martone ME, Ellisman MH, Kane CM. The cell: an image library-CCDB: a curated repository of microscopy data. Nucleic Acids Res. 2013:41:D1241-1250.

67. Hatano A, Chiba H, Moesa HA, Taniguchi T, Nagaie S, Yamanegi K, TakaiIgarashi T, Tanaka H, Fujibuchi W. CELLPEDIA: a repository for human cell information for cell studies and differentiation analyses. Database: J Biol Databases and Curation. 2011;2011:bar046.

68. Vempati UD, Chung C, Mader C, Koleti A, Datar N, Vidovic D, Wrobel D, Erickson S, Muhlich JL, Berriz G, et al. Metadata Standard and Data Exchange Specifications to Describe, Model, and Integrate Complex and Diverse HighThroughput Screening Data from the Library of Integrated Network-based Cellular Signatures (LINCS). J Biomol Screen. 2014;19:803-16.

69. Bendall SC, Nolan GP, Roederer M, Chattopadhyay PK. A deep profiler's guide to cytometry. Trends Immunol. 2012;33:323-32.

70. Qian Y, Wei C, Eun-Hyung Lee F, Campbell J, Halliley J, Lee JA, Cai J, Kong YM, Sadat E, Thomson E, et al. Elucidation of seventeen human peripheral blood B-cell subsets and quantification of the tetanus response using a density-based method for the automated identification of cell populations in multidimensional flow cytometry data. Cytometry Part B, Clinical Cytometry. 2010;78 Suppl 1:S69-82.

71. Newell EW, Sigal N, Bendall SC, Nolan GP, Davis MM. Cytometry by time-offlight shows combinatorial cytokine expression and virus-specific cell niches within a continuum of CD8+ T cell phenotypes. Immunity. 2012;36:142-52.

72. Aghaeepour N, Finak G, Flow CAPC, Consortium D, Hoos H, Mosmann TR, Brinkman R, Gottardo R, Scheuermann RH. Critical assessment of automated flow cytometry data analysis techniques. Nature Methods. 2013;10:228-38.

73. Courtot M, Meskas J, Diehl AD, Droumeva R, Gottardo R, Jalali A, Taghiyar MJ, Maecker HT, McCoy JP, Ruttenberg A. flowCL: ontology-based cell population labelling in flow cytometry. Bioinformatics. 2015;31:1337-9.

\section{Submit your next manuscript to BioMed Central and we will help you at every step:}

- We accept pre-submission inquiries

- Our selector tool helps you to find the most relevant journal

- We provide round the clock customer support

- Convenient online submission

- Thorough peer review

- Inclusion in PubMed and all major indexing services

- Maximum visibility for your research

Submit your manuscript at www.biomedcentral.com/submit
() BioMed Central 